\title{
Controle De Rebobinadeira De Máquina Flexográfica Por Meio De Inversor De Frequência E Potenciômetro
}

Control Flexographic Rewinder Machine Through Frequency Inverter And Potentiometer

\author{
Angelo de Souza Nicola¹, Flavio Perego Fagundes², Luciano Galdino³
}

\begin{abstract}
1.Tecnólogo em Mecatrônica Industrial e graduando em Engenharia Mecatrônica pela Faculdade ENIAC. E-mail: angelo.nicola@hotmail.com.

2. Operador CNC - Cumins. Tecnólogo em Mecatrônica Industrial pela Faculdade ENIAC. Graduando em Engenharia Mecatrônica na Faculdade ENIAC. E-mail: f.perego.f@gmail.com

3. Professor de Física e Elementos de Máquina na Faculdade ENIAC. Mestre em Ciências Exatas e da Terra na área de Física Nuclear pela USP, especializado em Física pela USP e Licenciado em Matemática pela UNG. E-mail: lucianogaldino1@yahoo.com.br.
\end{abstract}

\section{Resumo}

A rebobinadeira é uma máquina que tem como função rebobinar etiquetas e rótulos, e esse presente artigo aborda alguns tópicos importantes, principalmente no que diz respeito à melhoria deste tipo de máquina. Devido a alguns problemas detectados como, falha na metragem no rebobinamento de etiquetas ou rótulos, constante manutenção no sistema de transmissão, dificuldade do operador no setup correto e, principalmente, dificuldade no sistema de controle da rebobinadeira, houve a necessidade de redimensionar alguns dispositivos e implantar outros novos, tanto elementos mecânicos (polia e correia) como eletroeletrônicos (inversor de frequência, contador digital, sensor e potenciômetro). No intuito de aumentar a qualidade e a produtividade da rebobinadeira, estudos aprofundados são necessários e o respectivo artigo apresenta as ferramentas adequadas no desenvolvimento do mesmo, assim como as equações e cálculos essenciais para o desenvolvimento projeto.

Palavras-chave: Rebobinadeira. Transmissão. Etiqueta.

\begin{abstract}
The winder is a machine whose function is to rewind labels and tags, and that this article covers some important topics, especially regarding the improvement of this type of machine. Due to some problems detected as failed film rewinding of labels or tags, constant maintenance of the transmission system operator difficulty in the correct setup and especially the difficulty of controlling the winder system, there was the need to resize some devices and deploy new ones, both mechanical elements (belt and pulley) and electronics (frequency inverter, digital timer, sensor and potentiometer). In order to improve the quality and productivity of the winder, detailed studies are needed and the respective article presents the appropriate tools in the development of the same, as are the equations and calculations essential for the development project.
\end{abstract}

Keywords: Rewinder, Transmission, Label. 


\section{Introdução}

O presente projeto tem como objetivo tornar uma máquina flexográfica com maior eficiência de produtividade, com melhor desempenho em qualidade e também facilitar a parte operacional para o funcionário que operará a mesma. A escolha do tema aqui proposto deuse devido a grande dificuldade que o operador encontra para determinar a quantidade a ser produzido, o que influencia no monitoramento da qualidade da etiqueta adesiva ou rótulo e as perdas dos mesmos. Algumas das causas que acarretam essas dificuldades são a não existência de um controle de velocidade e a pouca eficiência de sua transmissão.

Conforme o mercado foi sofrendo alterações, a rebobinadeira revisora, uma máquina muito utilizada no processo de fabricação de rótulos e etiquetas adesivas, não acompanhou essa evolução, pois o operador para determinar o comprimento para bobinar certo pedido, primeiramente necessita fazer uma amostra manual e, a partir desta amostra, levar ao equipamento e regular o fim do curso de parada do motor. Depois desse processo finalizado inicia-se a produção, porém outros fatores negativos no processo dificulta uma produção sequencial, pois o operador que rebobina o determinado rótulo ou etiqueta gerada no processo de uma máquina flexográfica, revisa a qualidade da etiqueta, e quando encontra alguma anomalia neste processo torna-se necessária a interrupção do equipamento para substituir aquela etiqueta ou rótulo danificado, neste momento o operador se encontra em dificuldades, pois no momento em que ele encontra o problema até a interrupção do equipamento para solução do ocorrido, perde-se uma grande quantidade de tempo devido a máquina só existir uma única velocidade, o que faz necessário o operador desfazer de um montante do qual já estava pronto.

Enfim, o objetivo técnico desse projeto é a instalação de um inversor de frequência devidamente parametrizado com potenciômetro para controle de velocidade, além de um contador de comprimentos em metros com parada programada, podendo assim banir do processo a necessidade da confecção da primeira amostra manual, já o controle de velocidade servirá como uma melhoria na qualidade, pois o operador, ao detectar um problema numa etiqueta ou rótulo, consegue rapidamente interromper o movimento da máquina, devido a existência do potenciômetro, corrigir o problema e continuar o processo com uma velocidade menor controlada, podendo assim verificar se o problema de impressão no processo anterior persistiu. Com a velocidade agora reduzida e controlada pelo operador a probabilidade de uma falha na revisão passará a ser muito menor. 
Outra alteração em busca da melhoria da máquina é com relação ao sistema de transmissão. Atualmente era realizado através de correias do tipo $\mathrm{V}$, exigindo uma maior manutenção devida perdas de deslizamento da correia além de um tempo de reparo muito alto. A proposta é de substituir por uma transmissão por correias e polias sincronizadas, o que proporcionará um melhor desempenho do equipamento e um aumento considerável em sua produtividade, além de tornar este equipamento simplificado no que diz respeito à operação, tornando desnecessária uma grande habilidade do operador responsável pelo equipamento.

\section{Dispositivos do processo}

$$
\text { Segundo Franchi (2008) muitos }
$$
equipamentos foram desenvolvidos para as mais diversas variedades de aplicações e setores industriais, e um dos equipamentos mais utilizados nesses processos, juntamente com o CLP, é o inversor de frequência, um equipamento dinâmico e de enorme versatilidade que permite o uso de motores de indução para controle de velocidade e torque em substituição aos motores de corrente contínua. $\mathrm{Na}$ Figura 1 observa-se a parte física externa do inversor de frequência.
Figura 1- Inversor de Frequência.

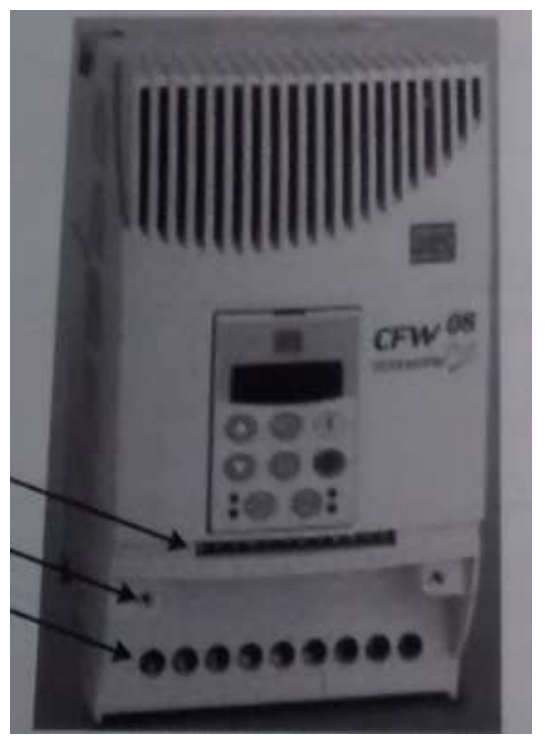

Fonte: Franchi, 2008, p. 19.

Capelli (2012) afirma que devido o baixo custo e a flexibilidade tecnológica do controle em corrente alternada, os motores CC junto aos seus respectivos acionamentos são considerados obsoletos. Com isso os acionamentos de motores elétricos em corrente alternada ocupam cerca de $80 \%$ dos sistemas de controle de motores elétricos, o que faz do inversor de frequência um dispositivo muito importante no setor industrial, podendo ser utilizado em outros setores também.

Segundo Capelli (2012) o inversor de frequência tem como função básica variar a velocidade de rotação de um motor $\mathrm{AC}$, e por isso depende da frequência da rede de alimentação. Quanto maior for a frequência, maior a rotação e vice-versa. Pode-se observar o que foi mencionado através da equação 1. 


$$
N=120 \times \frac{f}{P}
$$

Sendo:

$$
\begin{aligned}
& \mathrm{N}=\text { rotação em } \mathrm{RPM} \\
& \mathrm{f}=\text { frequência em } \mathrm{Hz} \\
& \mathrm{P}=\text { número de polos }
\end{aligned}
$$

Groover (2011) afirma que o sensor é um transdutor, ou seja, um dispositivo que converte uma variável física de uma forma em outra mais útil para a aplicação em questão.

De acordo com Capelli (2012) um dos componentes mais utilizados na área da eletroeletrônica são os sensores, e os mesmos estão presentes em diversos setores, como por exemplo, o setor industrial, predial ou até mesmo o setor comercial.

Nem sempre um sensor tem as características elétricas suficientes para poder ser utilizado em um sistema de controle. Geralmente o sinal de saída deve ser trabalhado antes de sua leitura no sistema de controle. Normalmente isso é realizado através de um circuito de interface que por sua vezproduz um sinal que possa ser lido pelo controlador. (THOMAZINI e ALBUQUERQUE, 2008, p. 17).

Existe uma grande quantidade de tipos de sensores e suas respectivas aplicações, sendo um dos mais utilizados na indústria, o sensor indutivo. Também chamados de sensores de proximidade, como o próprio nome diz são dispositivos capazes de identificar a aproximação de objetos metálicos, o que permite a substituição das chaves fim de curso (CAPELLI, 2012). Seu princípio de funcionamento é, aproximando-se um objeto metálico da face do sensor, consequentemente são induzidas correntes de Foucault. As perdas resultantes tiram energia do circuito oscilador, reduzindo assim as oscilações. A figura 2 representa este tipo de sensor. (THOMAZINI e ALBUQUERQUE, 2008).

Figura 2- Sensor Indutivo.

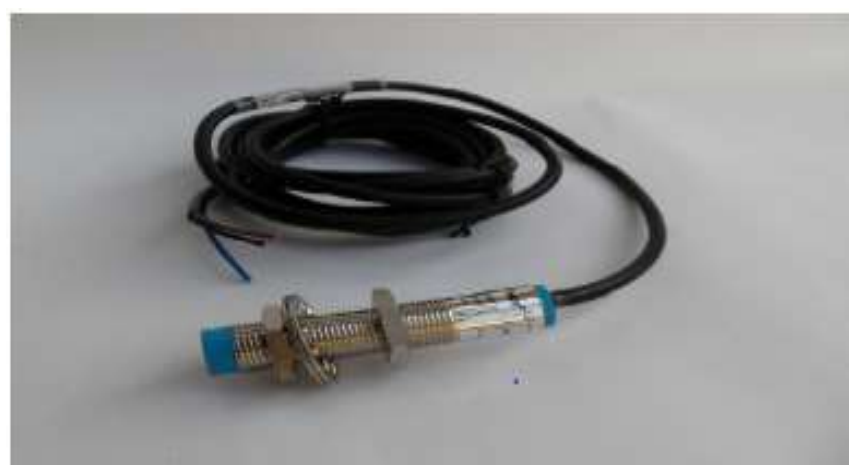

Fonte: Guia Rápido de Referência da Veder (2011)

$$
\text { Segundo Gussow (1997) os }
$$
potenciômetros são utilizados para variar ou mudar a quantidade de resistência de um circuito. Os potenciômetros normalmente possuem o elemento resistivo formado por carbono e o contato com o elemento resistivo fixo é por meio de um braço deslizante. À medida que o próprio braço deslizante gira, o respectivo ponto de contato com o elemento resistivo muda, é ai que a resistência entre o terminal do braço deslizante e os terminais da resistência fixa varia.

De acordo com Gussow (2009) os potenciômetros também podem variar a tensão 
aplicada a um circuito, como exemplo o circuito da Figura 3. A tensão de entrada é aplicada por meio dos terminais $\mathrm{AC}$ da resistência fixa, quando o terminal B que é o braço deslizante variar a sua posição, automaticamente a tensão de saída também vai variar. Quando o terminal B se aproximar em direção ao terminal A, a tensão de saída do circuito aumenta, já quando o mesmo vai em direção a $C$, a tensão de saída diminui.

Figura 3- Utilização de um potenciômetro para mudar a tensão.

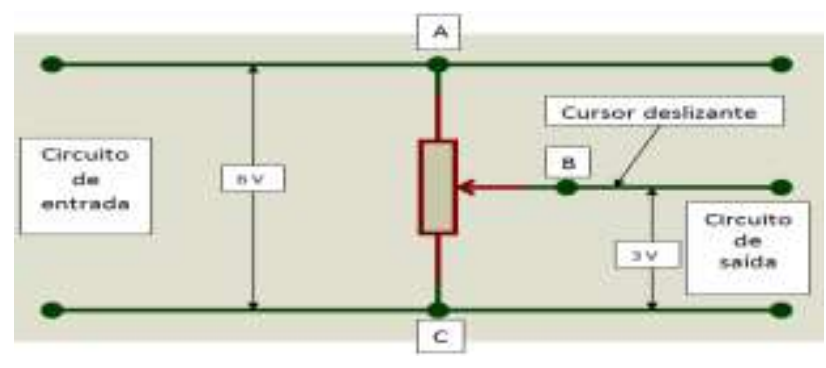

Fonte: Elaborado pelo autor.

De acordo com Groover (2011) um contador é utilizado para converter uma série de pulsos, em um valor digital que por sua vez é passado para o computador por um canal de entrada. Um dos tipos de contadores mais comum é o que conta pulsos elétricos, ele é constituído de portas lógicas sequenciais que recebem o nome de flip-flops, que são dispositivos eletrônicos com capacidade de memória e que podem ser utilizados no armazenamento dos resultados do processo de contagem.

Já Idoeta (2009) diz que contadores são circuitos digitais que variam os seus estados, através de um comando chamado clock, de acordo com uma sequência pré-determinada. Os mesmos são usados principalmente para contagens diversas, divisão de frequência, medição de frequência e tempo, geração de formas de onda e conversão de analógico para digital.

\section{Sistema De Transmissão (Motor, Polia E Correia)}

Segundo Cunha (2005) As transmissões por polias e correias constituem um recurso amplamente utilizado em vários segmentos industriais por sua versatilidade e seu baixo custo. Podendo ser usado nos mecanismos mais simples como em outros com certo grau de sofisticação.

Um dos componentes do sistema de transmissão por polia e correia são os motores. Neste estudo trata-se em específico dos motores trifásicos, que segundo Carvalho (2007) os mesmos são divididos em dois tipos: os assíncronos e os síncronos, os assíncronos são muito aplicados na indústria por se tratarem de motores robustos, de fácil manutenção e por serem de torque de partida alto o que atende a maioria das necessidades. Na figura 4 pode-se observar as partes externas que compõem o motor trifásico. 
Figura 4- Parte externa do motor.

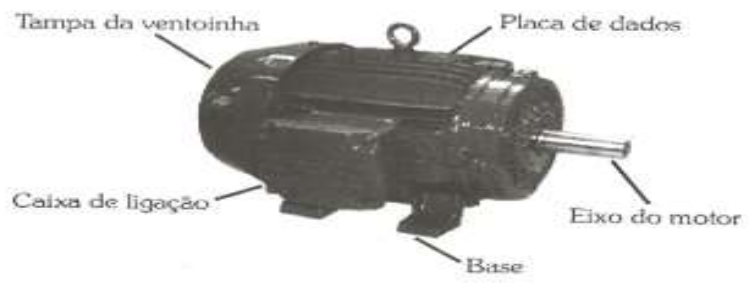

Fonte: Carvalho, 2007, p. 188.

$\mathrm{Na}$ parte frontal temos o eixo do motor que se usa para acoplamento mecânico. Na parte traseira temos uma tampa que protege contra o contato com a ventoinha do motor. A carcaça é fabricada em ferro fundido e em um dos lados tem-se a caixa de ligação, onde é realizada a ligação elétrica do motor. Segundo Carvalho (2007) a base do motor é de extrema importância, primeiro para sua fixação, e também por permitir determinar a potência do motor. Existem diversos tipos de carcaça, onde a escolha está ligada ao tipo de aplicação e como o motor será fixado à máquina.

Conforme Capelli (2012) o motor trifásico também é chamado de motor de indução pelo fato de as correntes de CA serem induzidas no circuito do rotor pelo campo magnético rotativo do estator.

Segundo Carvalho (2007) um motor sempre deve ter o torque suficiente a fim de movimentar uma carga, a equação 2 determina a potência em CV em função do torque.

$$
P(C V)=\frac{T(M . N) \times n}{7024}
$$

Carvalho (2007) mostra que para determinar a rotação com certa velocidade podemos aplicar a equação 3 .

$$
n=\frac{V}{\pi \times d}
$$

$\mathbf{V}=$ Velocidade em metros por minuto.

$\mathbf{d}=$ Diâmetro do eixo a qual é aplicada a rotação.

Na prática, para dimensionar um motor para uma devida aplicação outros dados relacionados à operação e instalação do motor devem ser considerados, um estudo mais profundo da aplicação é inevitável para a aplicação na fórmula. (CARVALHO, 2007).

Segundo Plaza e Janés (1995), polias são peças que tem o formato cilíndrico e giram em torno de um eixo, que juntamente com as correias e os motores formam um grupo que se completam, apesar de serem consideradas máquinas simples, as polias são muito úteis e nos proporciona diversas vantagens mecânicas. As polias assim como a maioria das peças mecânicas são divididas em algumas partes, conforme ilustrado na figura 5. As polias acopladas nos eixos dos motores recebem o nome de polia motora ou condutora, pois ela depende do motor para ser acionada já a polia movida é aquela que recebe movimento e força da polia motora e da correia. Os materiais usados nas construções das polias são: o material sintético, aços, liga leve como o alumínio e o bronze, 
porém o material mais utilizado na fabricação das polias é o ferro fundido.

Figura 5- Partes da polia.

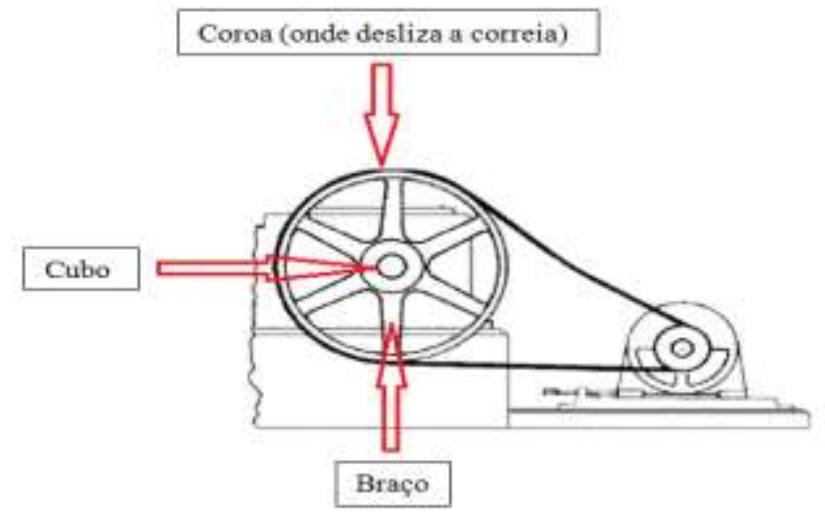

Fonte: Telecurso 2000.

Também chamada de roldana ou moitão a polia é utilizada nas indústrias em maquinários e equipamentos onde se é necessário à transmissão de movimento, velocidade, força e potência de um ponto para outro (PLAZA e JANÉS, 1995).

Segundo Budynas (2011), a polia dentada apresenta na sua superfície uma série de dentes que juntamente com as correias dentadas são engrenadas uma na outra sincronizando assim o movimento da polia com a correia (figura 6). Muito parecida com a engrenagem à polia dentada também conhecida como polia sincronizadora é normalmente requisitada quando existe a necessidade de não haver deslizamento nenhum.
Figura 6- Polia sincronizada com a correia.

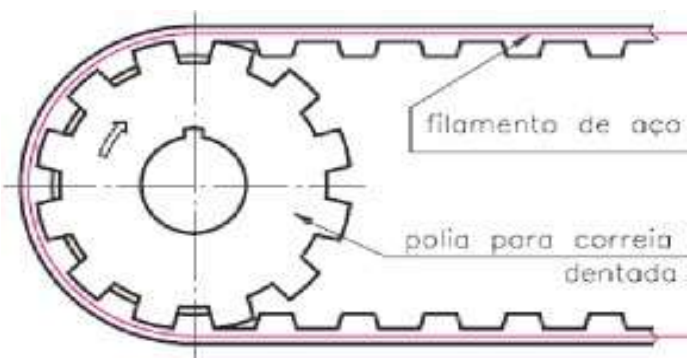

Fonte: Telecurso 2000.

As correias são elementos de máquinas que tem a função de manter o vínculo entre as polias e transmitir força (SENAI, 2009).

Cunha (2005) afirma que as correias podem se dispor de vários tipos e formatos, sejam elas:correias planas, trapezoidais simples e conjugadas, dentadas ou ranhuradas e transportadoras.

Segundo Manual Goodyear (s.d) as correias juntamente com as polias são um dos meios mais antigos de transmissão de movimento, este é um elemento flexível utilizado para transmissão de potência entre dois ou mais eixos. As transmissões por correias podem ser projetadas para grandes reduções ou multiplicações da velocidade, em uma mesma instalação de correias podemos obter variações de velocidades e para que isso seja possível basta instalar as correias, ora em um par, ora em outro par de rodas dentadas.

A correia dentada em uma união com a roda dentada permite uma transmissão de força sem deslizamento à força se transmite através dos flancos dos dentes e pode chegar $400 \mathrm{~N} / \mathrm{cm}^{2}$ o perfil dos dentes pode ser trapezoidal ou semicircular, este tipo de correia não alonga, não escorrega, não necessita de uma força pré- 
tensão, transmite potência a uma razão de velocidade constante, trabalha numa ampla gama de velocidades, eficiência entre $97 \%$ a $99 \%$ e não necessita lubrificação. (MANUAL GOODYEAR, s.d)

O Manual Goodyear afirma que para uma aplicação correta deve-se determinar o trabalho a ser realizado pela correia, assim determina-se o modelo da correia e através do modelo determina-se o passo do flanco dos dentes, o comprimento e largura de acordo com o dimensionamento para que a partir daí, possa determinar o passo da roda dentada.

A escolha do inversor de frequência para o projeto foi definido basicamente após cotações de preços em alguns fabricantes. O que levou a escolha do modelo IC5 do fabricante LS, pois o mesmo atende as necessidades do projeto e ainda oferece o melhor preço. Levando em consideração que no equipamento a ser automatizado (máquina flexográfica do modelo rebobinadeira revisora $160 \mathrm{~mm}$ ) já existe em funcionamento um motor de indução (WEG) com 1CV de potência e $1720 \mathrm{rpm}$ com alimentação em $380 \mathrm{~V}$. Vale ressaltar que o inversor de frequência utiliza uma entrada monofásica o que facilita a alimentação por se tratar de uma alimentação que não requer um serviço adicional da companhia de fornecimento de energia elétrica. Após dados retirados do motor, foi definido o inversor de frequência de $1 \mathrm{CV}$, entrada monofásica e saída trifásica.

Um dado importante na escolha do sensor a ser utilizado no respectivo projeto é a distância nominal de acionamento (Sn), o mesmo é encontrado na tabela de especificações dos fabricantes. Com o valor do Sn sendo multiplicado pelo fator de correção pode-se calcular a distância sensora operacional como se vê na equação 4.

$$
S a \leq 0,5 \times S n
$$

Onde:

Sa $=$ Distância sensora operacional;

$\mathbf{0 , 5}=$ Fator de correção;

Sn $=$ Distância nominal de acionamento

Aplicando a equação 4 ao respectivo projeto chega-se a distância sensora operacional que deve ser menor ou igual ao resultado, que neste caso é $2 \mathrm{~mm}$.

$$
\begin{gathered}
S a \leq 0,5 \times 4 \mathrm{~mm} \\
S a \leq 2 \mathrm{~mm}
\end{gathered}
$$

Com a distância sensora operacional calculada, obteve a distância necessária para o funcionamento indutivo do sensor com segurança. Isto é, a distância entre a face ativa (cabeça do parafuso) e a face do sensor. Com 
isso o sensor que atende as especificações do projeto é o TK-12N (P) (

O contador selecionado foi $\mathrm{O}$

Contador/Timer Microprocessado CB7, um contador fabricado pela Toki. Observa-se na equação 5 o cálculo do fator de multiplicação, que é o valor a ser inserido no contador para a parametrização do mesmo.

$$
P S=\frac{\pi \times d}{n}
$$

Onde:

PS = Fator de multiplicação.

$\mathbf{d}=$ Diâmetro do rolo onde está a polia com o sensor.

$\mathbf{n}=$ Número de pulsos na polia (correspondente ao número de parafusos na polia).

Se na rebobinadeira da máquina flexográfica, o diâmetro do rolo onde está a polia com o sensor é de $50 \mathrm{~mm}$. A polia tem 4 parafusos para contagem dos pulsos. Aplicando a equação 5, obtém-se o fator de multiplicação. Vale a pena ressaltar que o valor obtido deve ser dividido por 1.000 antes do mesmo ser inserido no contador, para que o valor a ser mostrado no display do contador esteja em metros, uma vez que no cálculo mostrado o valor do diâmetro do rolo onde está a polia com o sensor fora dado em milímetros.

$$
P S=\frac{\pi \times 50 m m}{4}=39,27
$$

$$
P S=\frac{39,27}{1000}=0,03927
$$

\section{Dimensionamento Das Polias}

Para substituir o sistema de transmissão de polias do modelo $\mathrm{V}$, foi retirado do equipamento a motora com uma especificação de $41 \mathrm{~mm}$ e as movidas sendo uma de $127 \mathrm{~mm}$ e outra com $57 \mathrm{~mm}$, a fim de manter o sistema de transmissão foi dimensionado o novo modelo de polias sendo elas do modelo $\mathrm{H}$ e o passo de 12,7mm. A equação 6 representa o diâmetro das polias em função do número de dentes.

$$
D=\frac{Z \times P}{\pi}
$$

Onde:

$$
\begin{aligned}
& \mathbf{D}=\text { Diâmetro da polia } \\
& \mathbf{Z}=\text { Número de dentes da polia } \\
& \mathbf{P}=\text { Passo da polia }
\end{aligned}
$$

Aplicando a equação 6 e utilizando os diâmetros das polias, chega-se aos valores do número de dentes das polias motora $\left(\mathrm{Z}_{1}\right), 1^{\mathrm{a}}$ polia movida $\left(Z_{2}\right)$ e $2^{a}$ polia também movida $\left(Z_{3}\right)$.

$$
41=\frac{Z_{1} \times 12,7}{\pi}
$$

$$
\begin{gathered}
Z_{1}=10,14 \quad \text { adotou }- \text { se } 10 \text { dentes } \\
127=\frac{Z_{2} \times 12,7}{\pi} \\
Z_{2}=31,41 \quad \text { adotou }- \text { se } 30 \text { dentes }
\end{gathered}
$$




$$
\begin{gathered}
57=\frac{Z_{3} \times 12,7}{\pi} \\
Z_{3}=14,12 \quad \text { adotou }- \text { se } 14 \text { dentes }
\end{gathered}
$$

\section{Investimento E Resultados}

O investimento total do projeto e a lista de materiais com seus respectivos preços encontram-se na Tabela 1.

Tabela 1: Lista dos materiais utilizados e preços.

\begin{tabular}{lc}
\hline Materiais & Preço \\
\hline Contador Digital & $\mathrm{R} \$ 180,00$ \\
\hline Inversor de Frequência & $\mathrm{R} \$ 500,00$ \\
\hline Potenciômetro & $\mathrm{R} \$ 40,00$ \\
\hline Sensor Indutivo & $\mathrm{R} \$ 60,00$ \\
\hline Polia Z=30 H & $\mathrm{R} \$ 50,00$ \\
\hline Polia Z=14 H & $\mathrm{R} \$ 50,00$ \\
\hline Polia Z=10 H & $\mathrm{R} \$ 50,00$ \\
\hline Correia H Sincronizada & $\mathrm{R} \$ 70,00$ \\
\hline
\end{tabular}

TOTAL DO INVESTIMENTO $\mathrm{R} \$ 1.000,00$

Fonte: Elaborado pelo autor.

Com um investimento de $\mathrm{R} \$ 1.000,00$ (um mil reais) conseguiu-se reduzir o tempo de setup de 15 minutos para 1 minuto, levando em consideração que o setup era realizado quatro vezes ao dia para atender a necessidade do cliente, lembrando que a rebobinadeira flexográfica tem capacidade de bobinar 40 metros de papel por minuto com um preço final de $R \$ 5,40$ (cinco reais e quarenta centavos) por metro.

Com tempo de máquina parada de 20 horas mensal para setup, antes da melhoria a perda de lucratividade girava em torno de $\mathrm{R} \$$ 6.400,00 (seis mil e quatrocentos reais) após a melhoria realizada o tempo de máquina parada para o setup gira em torno de1 hora mensal, ou seja, um ganho mensal de $\mathrm{R} \$ 6.076,00$ (seis mil e setenta e seis reais), com base nestes dados podemos afirmar que o investimento realizado foi pago em cinco dias trabalhados.

\section{Conclusão}

Com o desenvolvimento deste artigo pode-se perceber que a rebobinadeira de etiquetas, foi melhorada em vários aspectos, pois se conseguiu obter um ótimo resultado na parte de setup do equipamento. Conseguiu-se reduzir o tempo de cada setup eliminando a regulagem constante de um fim de curso que era realizada ao menos quatro vezes ao dia, pois agora basta introduzir a metragem em um contador programável que a operação é realizada com a qualidade que o cliente exige, além de eliminar os constantes ajustes com chaves Allen que deixava o operador em constante risco de acidente, pois além de realizar o setup, o modelo de transmissão exigia constantes ajustes para 
garantir a qualidade do produto. $\mathrm{O}$ novo modelo implantado com contador programável, inversor de frequência e o sistema de transmissão que passou a ser de correias sincronizadas, pode-se garantir um setup seguro, e as constantes manutenções que eram realizadas foram sanadas.

Por fim, com os argumentos aqui apresentados conclui-se que o projeto de automação da rebobinadeira flexográfica é viável e pode-se aplicar esse investimento não só nas grandes indústrias que utilizam esse tipo de equipamento, mas principalmente nas indústrias de pequeno porte, devido o investimento ser considerado pequeno e acessível, além de obter o retorno do investimento em um pequeno intervalo de tempo.

\section{Referências}

BUDYNAS, Richard G; NISBETTT J. Keith. Elementos de Máquinas de Shigley: Projeto de engenharia mecânica, $8^{\circ}$ Ed. Porto Alegre: AMGH, 2011, 1084 p.

CAPELLI, Alexandre. Automação Industrial: Controle do Movimento e Processos Contínuos. 2a Edição, São Paulo: Érica, 2012.

CARVALHO, Geraldo. Máquinas Elétricas. $2^{a}$ Edição rev., São Paulo: Érica, 2007.

CUNHA, Lamartine Bezerra. Elementos de Máquinas. Rio de Janeiro: LTC, 2005.
FRANCHI, Claiton Moro. Inversores de Freqüência: Teoria e Aplicações. $1^{a}$ Edição, São Paulo: Érica, 2008.

GUIA RÁPIDO DE REFERÊNCIA DA VEDER. Atualizado em (2011). Disponível em <www.veder.com.br> Acesso em: 24/11/2014.

GROOVER, Mikell P. Automação Industrial e Sistemas de Manufatura. $3^{a}$ Edição, São Paulo: Pearson, 2011.

GUSSOW, Milton. Eletricidade Básica. $2^{\mathrm{a}}$ ed. Porto Alegre: Bookman, 2009. 571 p.

GUSSOW, Milton. Eletricidade Básica. $2^{\mathrm{a}}$ ed. São Paulo: Pearson Makron Books, 1997. 639 p.

IDOETA, Ivan Veleije; CAPUANO, Francisco Gabriel. Elementos de eletrônica digital. $40^{\mathrm{a}}$ ed. São Paulo: Érica, 2009. 524 p.

MANUAL GOODYEAR - Correias de Transmissão de Potencia: Guia de Instalação, Manutenção e Solução de Problemas. Disponível em: http://www.goodyearep.com.br, acesso em 24/11/2014.

PLAZA \& JANÉS. Help Sistema de consulta interativa: ciência e tecnologia. São Paulo: Klick, 1995, 212p. 
TELECURSO

2000.

Curso

Profissionalizante: Elementos de Máquina -

volume 2. São Paulo: Globo. 2000, 256p.

TOMAZINI, Daniel; ALBUQUERQUE,

Pedro Urbano Braga de. Sensores Industriais:

Fundamentos e Aplicações. $5^{a}$ Edição

revisada. São Paulo: Érica, 2008.

225 NICOLA, A.S., FAGUNDES, F.P., GALDINO, L.: Controle De Rebobinadeira De Máquina Flexográfica Por Meio De Inversor De Frequência E Potenciômetro 